\title{
Differentiating Autism from Typical Development: Preliminary Findings of Greek Versions of a Pragmatic Language and Social Communication Questionnaire
}

\author{
Yvette Hyter $^{a}$ loannis Vogindroukas ${ }^{b}$ Evripidis-Nikolaos Chelas ${ }^{b}$ \\ Konstantinos Paparizos $^{\text {b }}$ Eleni Kivrakidou ${ }^{\text {b }}$ Vasiliki Kaloudi $^{\mathrm{b}}$ \\ a'Western Michigan University, Kalamazoo, MI, USA; ${ }^{b}$ Institute for Research and Education in Speech Therapy (IEEL), \\ loannia, Greece
}

\section{Keywords}

Pragmatics · Social communication · Autism · Assessment

\begin{abstract}
Objective: A social pragmatic communication disorder is one of the primary characteristics of children with autism. Although several measures of pragmatics and social communication exist, many are not comprehensive, do not examine the quality of interactions across contexts and interlocutors, and cannot be easily administered and interpreted. The aim of this article is to report on preliminary data collected using the Greek version of comprehensive social communication and pragmatic language questionnaires completed by parents and teachers interacting with young children in different contexts. Methods: A Greek translation of the social communication and pragmatic language questionnaires was administered to parents and teachers of 31 children diagnosed with autism and of 51 typically developing children aged 3.0-6.0 years. Analysis and Results: The performance on the Greek versions of the social communication and pragmatic language questionnaires of typically developing children was compared with the performance of the children with autism using a series of independent $t$
\end{abstract}

tests. Descriptive statistics were used to summarize participant characteristics and performances on the measure. Preliminary results showed that significant differences with moderate effect sizes were found between groups on $63 \%$ of the 30 test items. Conclusions: The performance of children on questionnaires may lead to the development of easily administered and interpretable assessments for differentiating children with pragmatics and social communication impairments, such as children with autism, from typically developing children.

(c) 2017 S. Karger AG, Basel

\section{Introduction}

One of the primary characteristics of autism is having difficulty with pragmatics and social communication [1]. Pragmatics is the use of language in contexts to convey meanings [2-4] and the cognitive, emotional, social perspectives of language use [5-7], which are influenced by culture and one's history of social practices [8]. Social communication refers to the symbiotic relationship among social cognition, executive functions, affective regulation, working memory, and pragmatics $[9,10$; Hyter and Sloane, unpubl. document]. Both pragmatics

\section{KARGER}

(c) 2017 S. Karger AG, Basel

E-Mail karger@karger.com

www.karger.com/fpl
Yvette Hyter, PhD, CCC-SLP Western Michigan University 1903 W. Michigan Ave.

Kalamazoo, MI 49008-5355 (USA)

E-Mail yvette.hyter@wmich.edu 
and social communication skills are necessary for successful interactions with others. If subjects exhibit poor pragmatics and social communication they may have difficulty in (a) communicating in a manner and with sufficient detail that the listener can understand and follow; (b) expressing their intentions; (c) monitoring the effect their behavior has on others; (d) initiating, following, maintaining, or making relevant contributions to a conversational topic; (e) changing their style of talking and conversational content to fit particular situations; (f) engaging in the retelling of past experiences; (g) explaining or describing events or objects; and/or (h) interpreting other intentions (e.g., differentiating joking from being serious) and nonverbal communication messages such as facial expressions (e.g., a look of discontent), vocal intonation (e.g., sarcasm), or body language (e.g., arms crossed and slumped in a chair) [11-15].

Since the publication of the DSM 5, there has been an increased focus on pragmatics and social communication, particularly on how these skills should be assessed, and how disordered pragmatics or social communication should be treated [16]. Assessing these skills is complicated because the nature of pragmatics and social communication dictates that assessments should take place in "natural contexts where these skills can be directly observed in real time" [16, p. 498]. There is a need for assessments of pragmatics and social communication that are contextualized, as well as reliable, valid, and evidenced based $[14,17]$.

The relationship between pragmatics and social communication focuses on communicators and the historical, social, and cultural contexts in which communication takes place $[8,12,15,18]$. These contexts (e.g., play, conversations with others, recounting past events, explaining something, listening to others) must be considered in any pragmatics and social communication assessment process [11]. Currently there are formal and informal measures for examining pragmatics and social communication [16, 17]. Formal assessment measures (standardized norm-referenced assessments) are difficult to use to examine the context and culture-bound skills of social communication and pragmatics, primarily because these formal assessments examine isolated skills or decontextualized behaviors $[8,14]$. For these reasons, the formal assessments are not able to be used to determine how a child would employ pragmatics and social communication skills in daily life. Such instruments include the Test of Pragmatic Language-2 (TOPL-2) [19] and the Comprehensive Assessment of Spoken Language (CASL) [20]. The TOPL-2 is organized to determine if the examinee

Differentiating Autism from Typical Development can make "judgements about decontextualized situations from another's perspective" [16, p. 510]. The CASL has two subtests that examine pragmatics and social communication, which focus on determining whether the examinee knows what to say or knows how to change what is said to be appropriate in given a situation [16]. Other formal assessments have a pragmatics checklist included as a subtest, such as the Diagnostic Evaluation of Language Variation [21], the Preschool Language Scales-5 [22], Bilingual English-Spanish Assessment [23], and the Test of Integrated Language and Literacy Skills [24].

Informal assessments, such as checklists and observations, are much more plentiful, and are frequently used to assess multiple components of pragmatics. A complete review of these assessments exceeds what is possible in this article, but a review is provided in a book chapter on pragmatic assessment and intervention by Hyter [16]. Such assessments include the Pragmatic Protocol [25], Discourse Skills Checklist [26], Systematic Observation of Communicative Interaction [27], Pragmatics Profile of Everyday Communication Skills in Children [28], Social Interactive Coding System [29], Parent Report Rating Scale [30], Targeted Observation of Pragmatics in Children's Conversation [31], Pragmatics Observational Measure [32], Communication and Symbolic Behavior Scales [33], Children's Communication Checklist-2 [34], Pragmatic Language Skills Inventory [35], and the Language Use Inventory [36].

Cordier et al. [17] called for more valid and reliable assessments that can be used to directly observe children in real-life contexts, and that can document changes in language across communication partners and contexts and inform intervention, as well as measure all components of social communication and pragmatics. As a preliminary effort to address the concerns raised by Cordier et al. [17], Hyter [9] and Hyter and Sloane [unpubl. document] have developed a comprehensive model of pragmatics and social communication. The foundation of the model is social cognition (i.e., theory of mind, perspective taking, intention reading), executive functions and its reciprocal component - affect regulation, pragmatic language (i.e., communicative functions, discourse management, presupposition skills), and working memory, conceptualized as what holds the aforementioned aspects of social communication together as a whole. Together these skills may facilitate one to make sense of others' points of view, adapt to diverse communicative contexts, be mentally flexible, hold on to information while processing other information, and implement and achieve communicative goals $[9,10]$. Affect dysregulation (the reciprocal compo- 
nent of executive functions) has the potential to hinder one's ability to engage effectively in the skills mentioned above [Hyter and Sloane, unpubl. document]. The aspects of pragmatics and social communication represented in this model comprise the components of the pragmatic language and social communication questionnaire used in this current study.

The goal of this study was to conduct a preliminary review of a pragmatic language and social communication questionnaire by comparing the performance of children with autism and those developing typically on questionnaires designed to examine pragmatic language and social communication in natural contexts [Hyter and Applegate, unpubl. document; Vogindroukas et al., unpubl. document]. It is our hypothesis that the children with autism would perform less well than the typically developing children in all domains examined with the questionnaires.

\section{Methods}

\section{Participants}

A total of 82 children (51 typically developing children and 31 children with autism) aged 3.0-6.0 years participated in this study. All participants with typical development were recruited through nursery schools in different cities in Greece. All participants with autism were recruited through speech-language therapists/pathologists (SLT/Ps) working for the national health system or in private practice in three different cities in Greece. Children with autism were assessed and diagnosed by a multidisciplinary team consisting of an SLT/P, child psychiatrist, occupational therapist, and special teacher who attended to the child in school. The inclusion criteria were as follows: (a) children with autism diagnosis had to be recruited through a national diagnostic center in Greece and (b) parents and teachers of typically developing children confirmed that the child did not have developmental delays or cognitive difficulties.

\section{Instrument}

Parent and teacher pragmatic language and social communication questionnaires are part of a larger assessment battery developed by Hyter and Applegate [unpubl. document]. These questionnaires are designed to be used by various responders (e.g., parents, teachers, SLT/Ps) in natural contexts (e.g., at home, preschool classroom) to observe the child interacting with multiple partners (adults and peers) in a variety of contexts. The questionnaires use a 5-point Likert scale ( 1 = hardly ever; $5=$ almost always $)$ and contain 30 (parent) and 35 (teacher) questions that address domains focused on child engagement (e.g., whether the child exhibits intersubjective awareness of others in his/her environment [37]), communication style (e.g., whether the child communicates primarily with words or gestures), communication functions (e.g., whether the child uses a range of speech acts), conversational discourse (e.g., whether the child engages in reciprocal conversations with others), executive functions (e.g., whether the child exhibits the ability to inhibit responses or to be flexible), social cognition (e.g., whether the child comments about others' feelings, thoughts, or perspectives), and perceptions (e.g., whether the child comments about others' experiential states) [Hyter and Applegate, unpubl. document]. Only the 30 questions in common on the parent and teacher forms were analyzed in this study.

Content validity was established by a panel of experts in speechlanguage pathology and education, who reviewed, eliminated, and refined items and determined their relevance to the constructs being assessed. The English version of these questionnaires was piloted in the US by preschool teachers, parents of typically developing preschool children, and graduate students enrolled in an SLT/P graduate program. The teachers and SLT/Ps provided preliminary feedback to researchers regarding the ease of use of the questionnaires, the ability of the questionnaires to accurately capture the social communication and pragmatic characteristics of the children in their care, and the usefulness of the information gleaned from the questionnaire in determining types of support required by the children. Based on the feedback received some questions were eliminated. The eliminated questions would have been difficult to measure because they required the responder (e.g., parent, teacher, SLT/P) to make a subjective decision about the child's intention, thoughts, or internal states. For example, the questions, "My child has negative feelings about others," and "My child prefers to interact with his peers," were modified to be more objective, or eliminated.

These questionnaires were designed to be used as school-wide screening instruments for young children (a) to provide feedback about the child's ability to function successfully in the classroom and determine whether support is required for the child to be able to participate fully in his/her preschool classroom, and/or (b) to determine whether a pragmatic language and social communication diagnostic assessment, and potentially intervention, is required [Hyter and Applegate, unpubl. document].

The questionnaires were translated from English into Greek by the second author (I. Vogindroukas). The questionnaires were then back-translated into English by the third author (E. Chelas) to certify linguistic or translation equivalence between the versions of the questionnaires. According to Peña [38], translated assessments must go beyond linguistic equivalence, to also include examinations of functional, cultural, and metric equivalencies. Functional equivalence ensures "that the instrument and elicitation method allow examination of the same construct" [38, p. 1,257]. The authors of the English and Greek version reviewed items on each questionnaire to determine whether the items were translated, using words that are familiar and understandable to parents and educators in Greece. This review yielded some items on each questionnaire that were framed differently than in the original English version. Cultural equivalence refers to "the way members of different cultural and linguistic groups view or interpret the underlying meaning of an item" [38, p. 1,258]. This assessment is currently underway with data from North America, Greece, and Brazil [39]. Metric equivalence is the "equivalence in item or question difficulty" [38, p. 1,259], resulting in the test "scores being comparable across cultures" [40, p. 123]. This level of equivalence has not yet been addressed between the two versions of the pragmatic language and social communication questionnaires.

\section{Procedures}

Ethical approval was obtained from the Western Michigan University Human Subjects Institutional Review Board in Kala- 
Table 1. Characteristics of participants

\begin{tabular}{lll}
\hline & $\begin{array}{l}\text { Typically developing } \\
\text { children }\end{array}$ & $\begin{array}{l}\text { Children } \\
\text { with ASD }\end{array}$ \\
\hline $\begin{array}{l}\text { Total per group, } n \\
\text { Gender, } n\end{array}$ & 51 & 31 \\
$\quad$ Male & 21 & 31 \\
$\quad$ Female & 30 & 0 \\
Mean age, months & 53.09 & 62.87 \\
\hline
\end{tabular}

ASD, autism spectrum disorder.

mazoo, MI, USA, and from the Organization for Social Protection and School Education of the City Council of Ioannina, Thessaloniki, and Athens. Moreover, all SLT/Ps that examined participants in the private sector had ethical approval from the PanHellenic Association of Logopedists. Ethical approval means that only participants whose parents provided consent and who gave assent to participate in the study were included in this research endeavor.

Parents (caregivers) and professionals (teachers or SLT/Ps) who had known the children or worked with the children for at least 3 months completed the questionnaires. All of those that completed the questionnaires observed the children engaged with or in the presence of adults and peers, and based their responses on what they had observed the child do at the time the questionnaire was being completed, and on the behaviors the child had been observed to demonstrate in the past. Time to complete the questionnaire varied from 5 to $15 \mathrm{~min}$.

\section{Psychometric Evaluation and Data Analysis}

A series of independent $t$ tests were conducted to compare pragmatic language and social communication skills as determined by parent and teacher questionnaires for typically developing children and children with autism. Only the questions in common on both the parent and teacher forms were compared between groups of participants. Descriptive statistics were used to summarize participant characteristics and performance on the measures used.

\section{Results}

Table 1 shows data on the age and ability of the participants. Of the typically developing children 30 (59\%) were female and $21(41 \%)$ were male. All the children with autism $(n=31)$ were male. The children with autism were a little more than 9 months older than the typically developing children.

A series of $t$ tests were implemented to compare the typically developing children and the children with autism on the 30 questions that the parent and teacher questionnaires had in common. An alpha level of 0.05 was used. Table 2 shows that there were significant differenc-
Table 2. Mean response of typically developing children compared with that of children with autism on each item on the parent and teacher questionnaires

\begin{tabular}{|c|c|c|c|}
\hline $\begin{array}{l}\text { Domains/ } \\
\text { item number }\end{array}$ & $\begin{array}{l}\text { Children with } \\
\text { autism }(n=31)\end{array}$ & $\begin{array}{l}\text { Typically developing } \\
\text { children }(n=51)\end{array}$ & $\begin{array}{l}\text { Effect } \\
\text { sizes }\end{array}$ \\
\hline \multicolumn{4}{|c|}{ Interactions with various partners and diverse contexts } \\
\hline $1^{\mathrm{a}}$ & 3.70 & $3.08^{* *}$ & 0.70 \\
\hline 2 & 3.93 & 4.31 & \\
\hline 9 & 3.42 & $4.51^{* *}$ & 1.06 \\
\hline 10 & 2.16 & $3.94^{* *}$ & 1.25 \\
\hline 29 & 3.67 & 4.10 & \\
\hline 30 & 2.93 & $4.16^{* *}$ & 1.02 \\
\hline \multicolumn{4}{|c|}{ Communication style } \\
\hline $3^{\mathrm{a}}$ & 2.28 & 2.25 & \\
\hline 4 & 4.33 & 4.71 & \\
\hline \multicolumn{4}{|c|}{ Communication functions } \\
\hline 5 & 3.97 & $4.53^{*}$ & 0.57 \\
\hline 6 & 4.35 & 4.65 & \\
\hline 7 & 3.74 & $4.74^{* *}$ & 0.78 \\
\hline 8 & 4.13 & $4.63^{* *}$ & 0.61 \\
\hline \multicolumn{4}{|c|}{ Conversational discourse } \\
\hline 12 & 2.50 & $3.98^{* *}$ & 1.12 \\
\hline 13 & 2.64 & $3.67 * *$ & 0.80 \\
\hline 14 & 2.42 & $4.14^{* *}$ & 1.34 \\
\hline 15 & 2.39 & $4.37^{* *}$ & 1.53 \\
\hline $16^{\mathrm{a}}$ & 1.77 & $2.80^{* *}$ & 0.80 \\
\hline 25 & 3.13 & $4.47^{* *}$ & 0.91 \\
\hline \multicolumn{4}{|c|}{ Executive functions } \\
\hline $11^{\mathrm{a}}$ & 2.00 & 1.61 & \\
\hline $23^{\mathrm{a}}$ & 2.26 & 1.96 & \\
\hline 24 & 3.39 & $4.10^{*}$ & 0.51 \\
\hline 26 & 2.56 & $3.31^{* *}$ & 0.62 \\
\hline $28^{\mathrm{a}}$ & 1.53 & 2.18 & \\
\hline \multicolumn{4}{|c|}{ Social cognition } \\
\hline 17 & 3.03 & 3.57 & \\
\hline 18 & 2.61 & $3.35^{*}$ & 0.55 \\
\hline 19 & 2.77 & 3.35 & \\
\hline 20 & 2.90 & $4.00^{* *}$ & 0.81 \\
\hline 27 & 1.93 & $4.10^{* *}$ & 1.58 \\
\hline \multicolumn{4}{|c|}{ Perceptions of self and others } \\
\hline $21^{\mathrm{a}}$ & 1.83 & $1.02^{* *}$ & 0.61 \\
\hline $22^{\mathrm{a}}$ & 1.65 & 1.82 & \\
\hline
\end{tabular}

es between groups with moderate to high effect sizes on $63 \%$ of the 30 items in the questionnaires.

Some of the questions are reverse scored (as indicated in Table 2); that is, the numerical scoring is inverted where the higher score is indicative of a concern regarding pragmatics and social communication. The reversescored questions 1, 16, and 21 showed statistically significant differences between groups. Question 1 is about 
a child's interactions with peers and adults. On this question, the children with autism (mean $=3.70$, SD 0.88) scored higher than the typically developing children (mean 3.08, SD 0.89), $t(79)=3.05, p=0.003$. These results indicate that the children with autism performed less well than the typically developing children when interacting with peers and adults. Question 16 focused on conversational discourse, specifically, whether the child presents as an assertive partner (e.g., initiates conversation, makes requests, asks for clarification, etc.) while being minimally responsive to his/her communication partner, as described by Fey [41]. Although both groups of children scored low on question 16, the typically developing children (mean $=2.80$, SD 1.17) scored significantly higher than the children with autism spectrum disorder (ASD; mean $=1.77, \mathrm{SD}=1.38), t(80)=-3.61, p=0.001$. These results suggest that the typically developing children were more likely to be initiators and less responsive to their communication partners than children with ASD. Question 21 focused on the child's perceptions of him-/herself and others, and yielded low scores for both groups. The children with ASD (mean $=1.83, \mathrm{SD}=1.55)$ expressed more negative opinions about themselves and others than did the typically developing children (mean $=1.02, \mathrm{SD}=$ 1.03), $t(79)=2.83, p=0.006$.

Excluding reverse scored items, typically developing children received higher scores on most of the pragmatic language and social communication questionnaire questions, particularly in the domains child engagement, communication functions, conversational discourse, and social cognition.

\section{Discussion}

This study examined 2 groups (typically developing children and children with ASD) of children between the ages of 3.0 and 6.0 on Greek versions of a social communication and pragmatic language questionnaire [5], which were translated, back-translated and adapted to the Greek language and culture. These data suggest that children with autism may exhibit more difficulty with pragmatic language and social communication when interacting with peers and adults in 4 of the 7 domains addressed by the questionnaires - interactions with multiple partners across distinct contexts, communicative functions, conversational discourse, and social cognition. Social interactions can support communicative development, increase quality of life, and increase prosocial behaviors $[42,43]$.
The children with ASD that participated in this study were equally included in play among their peers and positively regarded during play with peers. The children with ASD, however, were less likely to engage in pretend play. When we use "engage" in play, or "engagement," we are using this term as defined by Bloom and Tinker [37, p. 14], "emotional and social directedness for determining what is relevant for learning and the motivation for learning." In other words, engagement requires intersubjectivity (connectedness with others), social and emotional desire to connect with others, and shared meanings about others' intentions [44].

The typically developing children participating in this study demonstrated more abilities to express communicative functions (speech acts) than did the children with ASD, except for requesting actions of others. This finding is not surprising, as there is a history of research demonstrating that children with ASD use fewer communicative functions than their typically developing peers [45-47]. The children with ASD and those developing typically did not exhibit statistically significant differences in requesting actions.

The children with ASD were less likely to participate in conversational discourse than their typically developing peers. There is well-documented support that persons with ASD have difficulty engaging in conversations, including meeting Grice's maxim of quantity [48]. Persons with ASD exhibit difficulty in saying enough to engage in conversation, but not so much that one talks excessively, which affects turn taking, responsiveness to verbal and linguistic cues of conversational partners, and comprehension [13]. Children with ASD less often showed the ability to exhibit social cognitive skills, such as theory of mind, than their typically developing peers, but were as likely to demonstrate concern for others and to indicate how they were feeling about a situation or event.

What was not consistent with our original hypothesis was that the children with ASD and typically developing children would perform similarly on items related to conversational style (e.g., verbal vs. nonverbal communication), executive functions $[49,50]$, and perceptions of contexts, self, and others. On the Greek versions of the social communication and pragmatic language questionnaires, the typically developing children and those with autism showed no statistically significant differences in these areas. The questions about communication style focused on whether the children were primarily communicating verbally or nonverbally, e.g., communicating more with gestures and sounds. All of the children communicated more with words than nonverbally. Of the 5 ques- 
tions on the measure that focused on executive functions, only 2 were significantly different between both groups. These questions pertained to being flexible and engaging in self-calming behaviors. The other 3 questions dealt with executive functions referred to more extreme behavior issues such as getting into physical altercations with others, objecting to participating in daily activities, and responding negatively to new situations. One possible explanation for the similarities in scores of these groups of children is that the wording of the questions may have been perceived to convey willful behavior of the child, e.g., "My child objects to taking part in activities" [Hyter and Applegate, unpubl. document; question 11]. Even if a child with autism did have difficulty with new situations it may be unlikely for parents and teachers to attribute deliberate behavior to the child.

\section{Limitations}

There were several limitations to this preliminary analysis of data collected with a pragmatic and social communication measure on children with autism and children who were typically developing. First, this study included a small number of participants and there was no gender balance among the participants. Specifically only 31 of the participants were children with autism and all of these participants were males. Typically developing participants included a small number of females. Second, the analyses of data produced by the typically developing participants were not disaggregated based on gender. Specifically, the typically developing group of children included both males and females. Previous literature has shown that there may be gender differences for some pragmatic skills (e.g., communicative functions) [51]. Future studies with this preliminary pragmatic language and social communication questionnaire should include a balance of gender representation in both groups of participants. Third, this study shows a preliminary attempt of using the Greek version of a pragmatic and social communication measure. Additional analyses need to be con- ducted on the instrument including correlations among questions within each domain of pragmatics and social communication, and a factor analysis to determine if items cluster into dimensions/factors.

\section{Conclusions}

This study was a first attempt examination of the Greek version of a pragmatic language and social communication questionnaire. The results should be considered to be preliminary, and not yet generalizable to populations beyond the participants in this current study. The preliminary results suggest that the Greek version of the social communication and pragmatic language questionnaire may be able to differentiate children with ASD from those who are typically developing on abilities related to engagement with peers, communicative functions, conversational discourse, social cognition, mental flexibility, and self-calming behaviors.

\section{Acknowledgements}

We would like to extend our gratitude to the parents, teachers, and SLT/Ps who took time to complete the social communication and pragmatic language questionnaires. We thank Carly Roell, graduate student at Western Michigan University, who assisted with literature searches, and Nikolaos Petridis for the statistical analysis.

\section{Disclosure Statement}

The authors do not have financial conflicts of interest to disclose. Regarding nonfinancial disclosures, the authors are working together on the development and analysis of the Assessment of Pragmatic Language and Social Communication [APLSC; Hyter and Applegate, unpubl. document], the measure focused on in the study reported in this paper.

\section{References}

1 American Psychiatric Association: Diagnostic and Statistical Manual of Mental Disorders, ed 5. Washington, APA, 2013.

12 Adams C: Practitioner review: the assessment of language pragmatics. J Child Psychol Psychiatry 2002;43:973-987.

3 Bates E: Language and Context. New York, Academic Press, 1976.

Differentiating Autism from Typical Development
Folia Phoniatr Logop 2017;69:20-26 DOI: $10.1159 / 000479277$
Hart K, Fujiki M, Brinton B, Hart C: The relationship between social behavior and severity of language impairment. J Speech Lang Hear Res 2004;45:700-714.

7 Huang Y: Pragmatics, ed 2. Oxford, Oxford University Press, 2015.

8 Hyter YD: Pragmatic language assessment: a pragmatics-as-social practice model. Top Lang Disord 2007;27:128-145.
Prutting C, Kirchner D: A clinical appraisal of Hear Disord 1987.52:105-119. description. Semin Speech Lang 2005;26:1 188. 
9 Hyter YD: Complex trauma and prenatal alcohol exposure: clinical implications. American Speech, Language, Hearing Association SIG 16. Perspect School Based Issues 2012;13: $32-42$.

10 Timler GR: Social communication: a framework for assessment and intervention. ASHA Leader 2008; 13:10-13.

-11 Cordier R, Munro N, Wilkes S, Docking K: The pragmatic language abilities of children with ADHD following a play-based intervention involving peer-to-peer interactions. Int J Speech Lang Pathol 2013;5:429-440.

12 Cummings L: Pragmatic and Discourse Disorders: a Workbook. Cambridge, Cambridge University Press, 2015.

13 Green BC, Johnson KA, Bretherton L: Pragmatic language difficulties in children with hyperactivity and attention problems: an integrated review. Int J Lang Commun Disord 2014;49:15-29.

14 Norbury CF: Practioner review: social (pragmatic) communication disorder conceptualization, evidence and clinical implications. J Child Psychol Psychiatry 2014;55:204-216.

15 Perkins M: Pragmatic Impairment. Cambridge, Cambridge University Press, 2007.

16 Hyter YD: Pragmatic assessment and intervention in children; in Cummings L (ed): Research in Clinical Pragmatics: Perspectives in Pragmatics, Philosophy and Psychology. Cham, Springer International Publishing, 2017, pp 493-526.

17 Cordier R, Munro N, Wilkes-Gillan S, Speyer $\mathrm{R}$, Pearce WM: Reliability and validity of the Pragmatics Observational Measure (POM): a new observational measure of pragmatic language for children. Res Dev Disabil 2014;25: $1588-1598$.

18 Rivers KO, Hyter YD, DeJarnette G: Parsing pragmatics. The ASHA Leader 2012;17:1417.

19 Phelps-Terasaki D, Phelps-Gunn T: Test of Pragmatic Language, ed 2. Austin, Pro-Ed, 2007.

20 Carrow-Woolfolk E: Comprehensive Assessment of Spoken Language (CASL). Circle Pines, American Guidance Service, 1999.

21 Seymour HN, Roeper T, deVilliers JG: DELVNR (Diagnostic Evaluation of Language Variation) Norm-Referenced Test. San Antonio, The Psychological Corporation, 2003.

22 Zimmerman IL, Steiner VG, Pond RE: PLS-5: Preschool Language Scales. San Antonio, Pearson, 2011.

23 Peña ED, Gutierrez-Clellen V, Iglesias A, Goldstein B, Bedore L: Bilingual EnglishSpanish Assessment. San Rafael, Clinical Publications, 2014.
24 Nelson NW, Plante E, Helm-Estabrooks N, Hotz G: Test of Integrated Language and Literacy Skills. Baltimore, Brookes Publishing Co, 2016.

25 Prutting CA, Kirchner DM: A clinical appraisal of the pragmatic aspects of language. J Speech Hear Disord 1987;52:105-119.

26 Bedrosian J: An approach to developing conversational competence; in Ripich DN, Spinelli FM (eds): School Discourse Problems. San Diego, College Hill Press, 1985, pp 231255.

27 Damico JS: Clinical discourse analysis: a functional approach to language assessment; in Simon CS (ed): Communication Skills and Classroom Success: Assessment and Therapy Methodologies for Language and Learning Disabled Students. San Diego, College Hill Press, 1985, pp 165-204.

28 Dewart H, Summers S: The pragmatics profile of everyday communication skills in children, revised, 1995. http://complexneeds.org.uk/ modules/Module-2.4-Assessment-monitoring-and-evaluation/All/downloads/m08p080c/ the_pragmatics_profile.pdf (accessed March 20, 2017).

29 Rice ML, Sell MA, Hadley PA: The social interactive coding system (SICS): on-line, clinically relevant descriptive tool. Lang Speech Hear Services Schools 1990;21:2-14.

30 Girolametto L: Development of a parent report measure for profiling the conversational skills of preschool children. Am J Speech Lang Pathol 1997;6:25-33.

31 Adams C, Gaile J, Lockton E, Freed J: TOPICCAL applications: assessing children's conversational skills: turning a research instrument into a clinical profile. Speech Lang Ther Pract 2011;3:7-9.

32 Cordier R, Munro N, Wilkes-Gillan S, Speyer R, Pearce WM: Reliability and validity of the Pragmatics Observational Measure (POM): a new observational measure of pragmatic language for children. Res Dev Disabil 2014;35: 1588-1598.

33 Wetherby A, Prizant B: Communication and Symbolic Behavior Scales Profile. Baltimore, Paul H Brookes Publishing Co, 2001.

34 Bishop DVM: The Children's Communication Checklist-2. San Antonio, Psychological Corporation, 2006.

35 Gilliam JE, Miller L: Pragmatic Language Skills Inventory. Austin, Pro-Ed, 2006.

36 O’Neill DK: The language use inventory for young children: a parent-report measure of pragmatic language development for 18 to 47-month-old children. J Speech Lang Hear Res 2006;50:214-228.
7 Bloom L, Tinker E: The intentionality model and language acquisition: engagement, effort and the essential tension in development. Monogr Soc Res Child Dev 2001;66:1-91.

38 Peña ED: Lost in translation: methodological considerations in cross-cultural research. Child Dev 2007;78:1255-1264.

39 Hyter YD, Vogindoukas I, Fernandes F: Children with ASD: comparing parent and teacher responses on a social pragmatic communication questionnaire (poster). Am Speech Lang Hear Assoc, submitted, 2017.

40 Rubin A, Babbie ER: Research Methods for Social Work, ed 7. Belmont, Brooks/Cole, 2011.

41 Fey M: Language Intervention with Young Children. Austin, Pro-Ed, 1986.

42 Nijs S, Maes B: Social peer interactions in persons with profound intellectual and multiple disabilities: a literature review. Educ Train Autism Dev Disabil 2014;49:153-165.

43 Yuill N, Strieth S, Roake C, Aspden R, Todd $B$ : Brief report: designing a playground for children with autism spectrum disorders - effects of playful peer interactions. J Autism Dev Disord 2007; 17:1192-1196.

44 Gerber S: A developmental perspective on language assessment and intervention for children on the autistic spectrum. Top Lang Disord 2003;23:74-94.

45 Casenhiser DM, Binns A, McGill F, Morderer O, Shanker SG: Measuring and supporting language function in children with autism: evidence from a randomized control trial of a social-interaction-based therapy. J Autism Dev Disord 2015;45:846-857.

46 Dennis M, Lazenby A, Lockyer L: Inferential language in high-functioning children with autism. J Autism Dev Disord 2001;31:47-54.

47 Tager-Flusberg H, Anderson M: The development of contingent discourse ability in autistic children. J Child Psychol Psychiatry 1991; 32:1123-1134.

48 Grice HP: Logic and conversation; in Cole P, Morgan J (eds): Studies in Syntax and Semantics III: Speech Acts. New York, Academic Press, 1975, pp 183-198.

49 Bishop DVM, Norbury CF: Executive functions in children with communication impairments, in relation to autistic symptomatology I: generativity. Autism 2005;9:7-27.

50 McCloskey G, Perkins LA, Van Divner B: Assessment and Intervention for Executive Function Difficulties. New York, Routledge/ Taylor \& Francis Group, 2009.

51 Hwa-Froelich D, Kasambira D, Moleski A: Communication functions of African American Head Start children. Commun Disord Q 2007;28:77-91. 